\title{
Doubts about ventricular reconstruction
}

$\mathrm{T}$ he rationale for ventricular reconstructive surgery in patients with left ventricular dysfunction caused by coronary artery disease is that the excision of scar tissue and reduction of left ventricular volume could improve mechanical function. The procedure has been advocated strongly by some investigators (Dor, V. Heart Fail. Rev. 6, 187-193 [2001]), but the limitations of this approach have now been highlighted in a paper published in the New England Journal of Medicine. The Surgical Treatment for Ischemic Heart Failure (STICH) Hypothesis 2 investigators report that CABG surgery with left ventricular reconstruction does not improve 2 year survival or rates of cardiac hospitalization in patients with coronary artery disease when compared with CABG alone (Jones, R. H. et al. N. Engl. J. Med. 360, 1705-1717 [2009]). The lack of clinical benefit for ventricular reconstruction in this study is disappointing, but raises some important questions, which can be addressed by considering the study and the procedure itself in more detail.

The STICH trial was a multicenter, international, randomized study sponsored by the National Heart, Lung, and Blood Institute. The investigators enrolled 1,000 CABG-eligible patients with coronary artery disease and anterior left ventricular dysfunction (left ventricular ejection fraction $<35 \%)$. Patients with recent myocardial infarction, those who were due to undergo percutaneous coronary intervention or aortic valve replacement, and those with a life expectancy less than 3 years were excluded from the study. Patients who were not eligible for medical therapy alone (left main coronary artery stenosis $\geq 50 \%$ or Canadian Cardiovascular Society class III or IV angina), but who were candidates for ventricular reconstruction, were randomized within the STICH Hypothesis 2 program. Data from patients eligible for medical therapy with or without CABG surgery, but not for ventricular reconstruction are being analyzed in the ongoing STICH Hypothesis 1 arm of the trial.

Although ventricular reconstruction significantly reduced ventricular volume, the procedure was not associated with any discernable clinical benefit in terms of mortality, cardiac hospitalization, or cardiovascular symptoms when compared with CABG surgery alone. Moreover, the use of ventricular reconstruction increased the duration and financial costs of both surgery and hospitalization, but it did not lead to an increase in surgical mortality. Importantly, patients enrolled in the STICH trial did not have ventricular aneurysm; it is important to distinguish between left ventricular reconstructive surgery in the absence of aneurysm and left ventricular aneurysmectomy, which has been demonstrated to attenuate clinical symptoms (Mickleborough, L. L. et al. J. Thorac. Cardiovasc. Surg. 128, 27-37 [2004]).

Why did ventricular reconstructive surgery fail to demonstrate any clinical benefit in the STICH study? One intriguing possibility is that the procedure, which entails resection of the hypokinetic/akinetic region of myocardium, disturbs the three-dimensional architecture of the left ventricle to an extent that has, thus far, been underappreciated. A number of investigators have described a helical structure and a spiral pattern of contraction and relaxation in the myocardial muscle. Radionuclide angiocardiography, echocardiography, and MRI confirm that the normal mechanical function of the left ventricle is a consequence not only of myocardial shortening, lengthening, narrowing, and widening, but also of a spiral twisting motion (counterclockwise and clockwise). Ventricular reconstructive surgery might, therefore, disrupt the coordinated helical structure of the myocardium in a manner that compromises both systolic and diastolic function. Furthermore, as the STICH investigators discuss in their paper, it is possible that reconstructive surgery impairs diastolic filling to the extent that the physiological benefits of reduced wall stress and improved systolic ventricular function are negated. In addition, selection bias-specifically, the possibility that patients who were offered ventricular reconstructive surgery directly differed substantially from those enrolled in the trial-could have influenced the results of this study.

The conclusion of the STICH Hypothesis 2 trial-that ventricular reconstructive surgery does not improve cardiac morbidity or mortality at 2 years follow-uphighlights the importance of the three-dimensional structure of the left ventricle in systolic and diastolic function. With the evolution of sophisticated imaging techniques, we should begin to investigate the architecture of the cardiac chambers so that, in the future, myocardial structure can be assessed systematically before and after interventions aimed at restoring left ventricular function.

doi:10.1038/nrcardio.2009.78
V. Fuster is the Editorin-Chief of Nature Reviews Cardiology.

Competing interests The author declares no competing interests. 